\title{
Analysis of radiating structures placed on multilayer dielectric
}

\author{
M. Wnuk \& M. Bugaj \\ Faculty of Electronics, Military University of Technology, Poland
}

\begin{abstract}
In this work, a solution to the problem of electromagnetic fields scattering on periodical, limited, planar antenna structures, placed on the border of two dielectric layers is formulated. One led out of scattering this kind of structure and defined in its generalization for the case of antenna with a multilayered dielectric basis. By using Galerkin's spectral method the problem was brought to an arrangement of algebraical equations with disposition on coefficients of the current's disposition on metal elements of an antenna arrangement, with regard to disposition of field on Floquet's harmonics. The complete transverse dimension of an antenna was taken into account by introducing antenna's window functioning solution of unlimited antenna. The formalism allows the modeling of periodical actions, dielectrics, and composite antenna arrays.

Keywords: Antenna arrays, multilayer dielectric, Green function.
\end{abstract}

\section{Introduction}

Antenna arrays usually consist of periodical metal structures placed on, or set in, multilayer dielectric. Changes of size or shape of each of the antenna's elements allows for effective modelling of spectral characteristics of parameters of antenna array. These changes can also be modelled by the selection of geometrical and physical parameters of dielectric layer surface and coverage.

In the case of big transversal dimensions of antenna (100 lengths of wave) and several dozen or more single radiating metal elements, characteristics of antenna radiation in good approach respond to characteristics of an unlimited antenna structure.

When the condition of fulfilling periodicality of such an antenna is fulfilled and it is excited by a flat wave with linear and homogenize module faze, the problem of diffraction of such a structure is brought to an analysis of a single 
elementary antenna cell. For structures of smaller shapes and fewer numbers of elements, effects connected with their limitation, especially boundary effects occurring on external cells can have an influence on characteristics of antenna work and should be taken into account during modelling them.

So far, a strict method of solving the problem of diffraction on periodical limited structure is not known. Attempts to directly solve the question, with direct usage of spectral moment method, are only formally strict, because their exactitude is limited by limitations of dimensions of matrixes used in procedures of solving them $[\mathrm{L}-3,4,5]$.

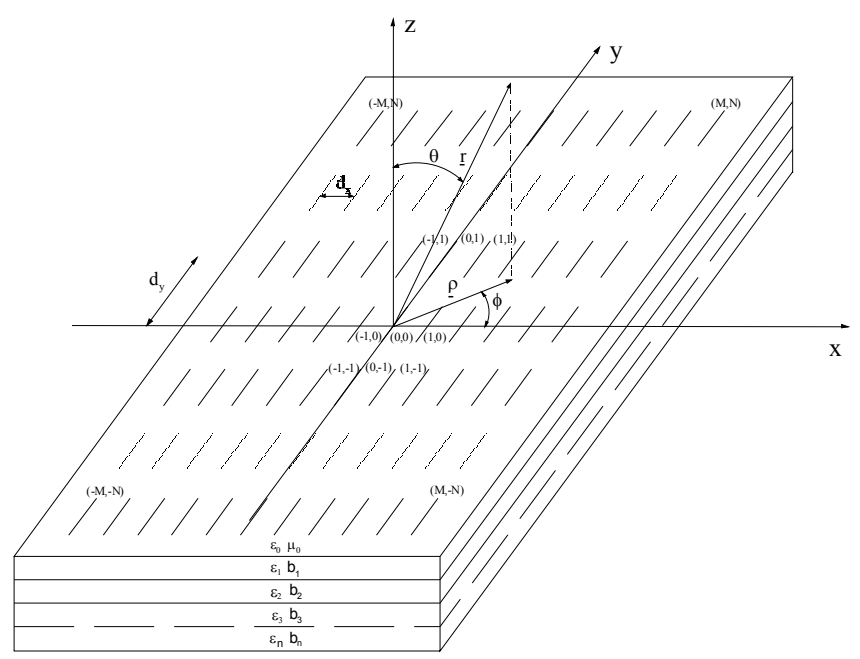

Figure 1: $\quad$ Planar periodic multilayer antenna structure.

Nevertheless, for antenna arrays consisting of several or several dozen of basic cells of regular shape, this method gives good results. Other known methods of solving problems of diffraction on larger periodical structures, such as modifications mentioned in the above method by limiting the number of input base functions and accurate selection of their course [L-1] or iteration method used in solution by sequenced usage of fast Fourier transform are approached, and can be used only in some simple, easy to analyse cases.

The analysis presented is based on the idea of window function [L-2] used in seeking current distribution and composed in the structure of a problem of diffraction on periodical unlimited structure. Presented in the paper is the sequence of the next steps of solving leads to strict solution and takes into account only predicted difficulties with convergence of the used procedure.

\section{Diffraction of electromagnetic field on limited periodical antenna array}

We consider a case with limited antenna array periodical in plane $z=0$ in direction of $\mathrm{x}$ and $\mathrm{y}$ "axis". 
Antenna array, as stated before, can be set in any unlimited multilayer dielectric in planes perpendicular to axis $\mathrm{z}$ or placed on border of two layers of dielectric. For a simpler analyse we divide the antenna surface in plane $\mathrm{z}=0$ on identical cells (Fig. 2) numbered with integer numbers $(m, n)$, where the central point $\left(\left(\mathrm{x}_{0}, \mathrm{y}_{0}\right)\right.$ of the central cell $(0,0)$ is placed at the beginning of coordinate system $\mathrm{x}=0, \mathrm{y}=0$, and points $\mathrm{x}=\mathrm{x}_{\mathrm{m}}, \mathrm{y}=\mathrm{y}_{\mathrm{n}}$ are central points of next cells $(\mathrm{m}, \mathrm{n})$.

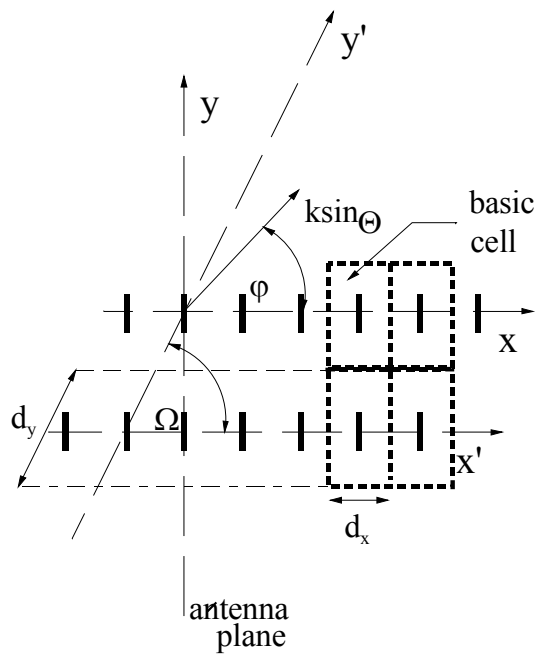

Figure 2: $\quad$ Partition of the antenna surface on cells.

Antenna cells with carrier function $\mathrm{h}_{\mathrm{mn}}$ cover the part of plane $\mathrm{z}=0$ responding

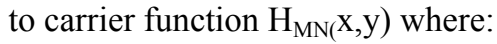

$$
\begin{aligned}
& H_{M N}(x, y)=1, \text { dla } x \in\left[-M d_{x}, M d_{x}\right] i y \in\left\lfloor-N d_{y}, N d_{y}\right\rfloor, \\
& H_{M N}(x, y)=0, \text { dla } x \notin\left[-M d_{x}, M d_{x}\right] l u b y \notin\left[-N d_{y}, N d_{y}\right]
\end{aligned}
$$

Integer numbers $m, n$ number cells in direction of axis $x \quad m=-M$,$M+1, \ldots, 0, \ldots, M-1, M$ and towards axis y $n=-N,-N+1, \ldots, 0, \ldots, N-1, N$. Metalized elements are placed periodically on the same limited area of plane $z=0$ in each cell of antenna array $(m, n)$ and cover the area of carrier function $A_{M N}(x, y)$ :

$$
\sum_{m, n} a_{m n}(x, y)=A_{M N}(x, y)
$$

Let's notice that functions $H_{M N}(x, y), A_{M N}(x, y)$ are decomposed on components dependent from $\mathrm{x}$ and $y$ :

$$
H_{M N}(x, y)=H_{M N x}(x) H_{M N y}(y),
$$




$$
A_{M N}(x, y)=A_{M N x}(x) A_{M N y}(y) .
$$

Because of the limitation of the antenna in plane $\mathrm{z}=0$ antenna's cells are distinguished, by their placement towards the beginning of coordinate system $(x, y)=(0,0)$. However, in this case the solution (current distribution) is changing from cell to cell. In a periodical unlimited system each cell $(m, n)$ can be a central cell. In a limited periodical system the central cell is univocally defined by its placement in the centre area of the antenna array. Every other cell is univocally defined by placement of its centre towards the centre of the array area $(x, y)=(0,0)$.

For further consideration it is necessary to define window carrier function $H_{M N m n}(x, y)$ of the antenna array. $H_{M N m n}(x, y)$ functions change themselves depending on in which cell a hypothetical beginning of coordinate system has been placed and are defined by vector translation $\left(x_{m}, y_{n}\right)=\left(m d_{x}, n d_{y}\right)$ of function $H_{M N}$ :

$$
H_{m n}(x, y)=H_{00}\left(x-x_{m}, y-y_{m}\right)=H_{M N}\left(x-x_{m}, y-y_{m}\right) .
$$

Their Fourier transforms equal respectively:

$$
\begin{gathered}
F\left(H_{00}\right)\left(k_{x}, k_{y}\right)=\frac{M N d_{x} d_{y}}{\pi^{2}} \frac{\sin M k_{x} d_{x}}{M d_{x}} \frac{\sin N k_{y} d_{y}}{N d_{y}}, \\
\bar{H}_{m n k} \equiv \bar{H}_{M N 00}\left(k_{x k}, k_{y k}\right)=\left(d_{x} d_{y}\right)^{-1} F\left(H_{00}\right)\left(k_{x k}, k_{y k}\right)= \\
=\frac{M N}{\pi^{2}} \frac{\sin M k_{x k} d_{x}}{M d_{x}} \frac{\sin N k_{y k} d_{y}}{N d_{y}}, \\
\bar{H}_{m n k} \equiv \bar{H}_{m n}\left(k_{x k}, k_{y k}\right)=\bar{H}_{00}\left(k_{x k}, k_{y k}\right) \cdot \\
\exp \left[-i\left(m k_{x k} d_{x}+n k_{y k} d_{y}\right)\right]=\bar{H}_{o 0}\left(k_{x k}, k_{y k}\right) \exp \left[-i\left(m \varphi_{x k}+n \varphi_{k y}\right)\right] .
\end{gathered}
$$

So, as we see Fourier transforms of the following cells experience phase modulation which equals to respective Floquet harmonic.

\section{Solving the problem of electromagnetic field diffraction on antenna array with limited dimensions}

Formulating the problem of diffraction by defining matrixes of diffraction antenna array in spectral space, allows for direct introduction of window function to make a solution for unlimited case. [L-11]. In such a case only equations containing current distribution are modified: 


$$
\begin{aligned}
& \underline{J}(x, y)=H_{m n}(x, y) \sum_{q} \underline{j}_{q} \underline{\varphi}_{q}(x, y)=\sum_{k} \overline{\bar{J}}_{k} \bar{H}_{m n k} e_{k}(x, y), \\
& \underline{E}(x, y, 0)=\left(\underline{\underline{G}} * \underline{J} * H_{m n}\right)(x, y)=\sum_{k} \overline{\underline{G}}_{k} \overline{\bar{J}}_{k} \bar{H}_{m n k} e_{k}(x, y)
\end{aligned}
$$

It is obtained by multiplying amplitudes of their spectral representations by Fourier transform of window function $\bar{H}_{m n k}$ responding to selected cell $(m, n)$. Another form of window function $H_{m n}(x, y)$ responds to each cell $(m, n)$ because of its placement towards the central carrier of window function $H_{M N}(x, y)$. For each couple of numbers $(m, n)$ another solution determining current distribution on the metalised part of cell $(m, n)$ is calculated. .

From the formal point of view, formulating and solving the problem of diffraction for an antenna array with limited dimensions should take into account the continuous spectrum of the value of the wave number $k$ in spectral distribution of parameters field and currents. It means that one should use continuous Fourier transform instead of spectral distributions in those points which respond to discreet values of wave numbers of the following Floquet harmonics. The numerical solution of such a problem also in this case would bring the analysed problem to a problem with discreet spectrum used by numerical procedures such as fast Fourier transform (FFT). This is why it seems reasonable to use spectral distributions of analysed values with spectrum determined by harmonics responding to a case with unlimited periodical structure.

So when concerning formal note of equations, except for modifications in the above equations by introducing a window function, formulating and solving presented problem of diffraction on unlimited periodical antenna structure is obtained by distribution of base functions, currents and fields on Floquet harmonics, in a way similar to unlimited antenna array:

$$
\begin{gathered}
\underline{\varphi}_{q}(x, y)=\sum_{k} \underline{\varphi}_{q k} \underline{\Psi}_{k}(x, y), \\
\underline{J}(x, y)=\sum_{q, k} \underline{j}_{q} \bar{\varphi}_{q k} \bar{H}_{m n k} e_{k}(x, y), \\
\underline{E}_{t}^{i}(x, y, 0)=\sum_{k} v_{k}^{i} \underline{\Psi}_{k}(x, y), \\
\underline{E}_{t}^{e}(x, y, 0)=\sum_{k}\left(1 \pm \Gamma_{k}\right) v_{k}^{i} \underline{\Psi}_{k}(x, y), \\
\underline{E}_{t}^{s}(x, y, 0)=\sum_{k} v_{k}^{s} \underline{\Psi}_{k}(x, y),
\end{gathered}
$$

by solving diffracted field with inducted currents, with usage of Green function 


$$
\underline{E}_{t m n}^{s}(x, y, 0)=\sum_{k} \bar{G}_{k} \bar{J}_{k} \bar{H}_{m n k} e_{k}(x, y)
$$

and throwing on, with usage of the Galerkin method, inducted currents condition of resetting total field on surface of metal antenna elements:

$$
\underline{E}_{t m n}(x, y, 0) A_{M N}=\left(\underline{E}_{t}^{e}(x, y, 0)+\underline{E}_{t m n}^{s}(x, y, 0)\right) A_{M N}=0
$$

As a result we obtain system with double matrix algebraically equations:

$$
\underline{\underline{W}} \underline{\underline{j}}=\underline{V^{s}} \quad \underline{\underline{N V^{i}}}=-\underline{\underline{U}} \underline{\underline{j}}
$$

where:

$$
\begin{aligned}
& \underline{\underline{W}]_{k q j}} \equiv W_{k q j}=\sum_{i} \psi_{k i}^{*} \bar{G}_{k i j} \bar{\varphi}_{q k j} \bar{H}_{m n k} \\
& \underline{\underline{N}}]_{q k j} \equiv N_{q k j}=\left(1+\Gamma_{k}\right) \bar{\varphi}_{q k j}^{*} \psi_{k j}, \\
& \underline{\underline{U}]_{p q i j}} \equiv U_{p q i j}=\sum_{k} \bar{\varphi}_{p k i}^{*} \bar{G}_{k i j} \bar{\varphi}_{q k j} \bar{H}_{m n k} \\
& {[j]_{q j} \equiv j_{q j},} \\
& \left\lfloor V^{s}\right\rfloor_{k} \equiv v_{k}^{s} \text {, } \\
& \left\lfloor\underline{V}^{i}\right\rfloor_{k} \equiv v_{k}^{i} \text {. }
\end{aligned}
$$

Solution of these equations gives coordinates of current distribution:

$$
\begin{aligned}
{[j]_{q x m n} } & \equiv j_{q x m n}, \\
{[j]_{q y m n} } & \equiv j_{q y m n},
\end{aligned}
$$

in a cell defined by indexes of window function $H_{m n}$

Solving the above system of algebraical equations for the next window functions $H_{m n}$, we obtain current distribution $J_{m n}$ on next cells $(m, n)$ of limited periodical antenna array, so we obtain $M x N$ solutions of the above system of equations for $M x N$ courses of functions $H_{m n}(x, y)$. Summarized current distribution on all cells at the same time can be presented in a following form: 


$$
\underline{J}(x, y)=\sum_{m, n} \underline{J}_{m n}(x, y) a_{m n}(x, y)
$$

where:

$$
\begin{aligned}
& \underline{J}(x, y)=\sum_{q} \underline{j}_{q} \underline{\varphi}_{q}(x, y)= \\
& =\sum_{k} \underline{\bar{J}}_{k} e_{k}(x, y)=\sum_{k} \sum_{q} \underline{j}_{q} \bar{\varphi}_{q k} e_{k}(x, y)
\end{aligned}
$$

However, the final solution needs univocal connection of current distribution with raining and diffracted field.

$$
\underline{V}^{s}=\underline{\underline{W}} \underline{\underline{N}} \underline{\underline{N}} \underline{\underline{V^{i}}}
$$

equivalent to construction of matrix of diffraction of antenna structure:

$$
\left[\begin{array}{l}
\underline{\mathrm{V}}^{S+}+\underline{\underline{\Gamma}} \underline{V}^{i-} \\
\underline{\mathrm{V}}^{s-}-\underline{\underline{\Gamma}} \underline{V}^{i+}
\end{array}\right]=\left[\begin{array}{ll}
\underline{S} 11 & \underline{\underline{S}} 12 \\
\underline{S} 21 & \underline{S} 22
\end{array}\right]\left[\begin{array}{l}
\underline{V}^{i-} \\
\underline{V}^{i+}
\end{array}\right]
$$

so it needs one univocal solution of above system of equations, which takes into account current distributions on all cells of antenna at the same time.

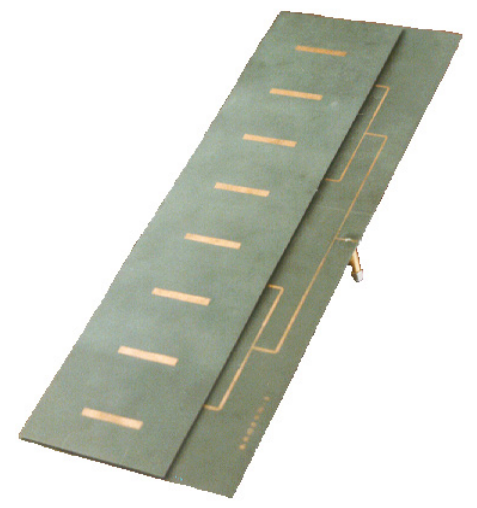

Figure 3: $\quad$ Eight element antenna array. 
Calculations have been made for a structure presented in fig. 3. Obtained results of calculations with comparison to the made measurement are presented in fig.4 although the time of calculations is very long, they are encouraging to further work in the direction.

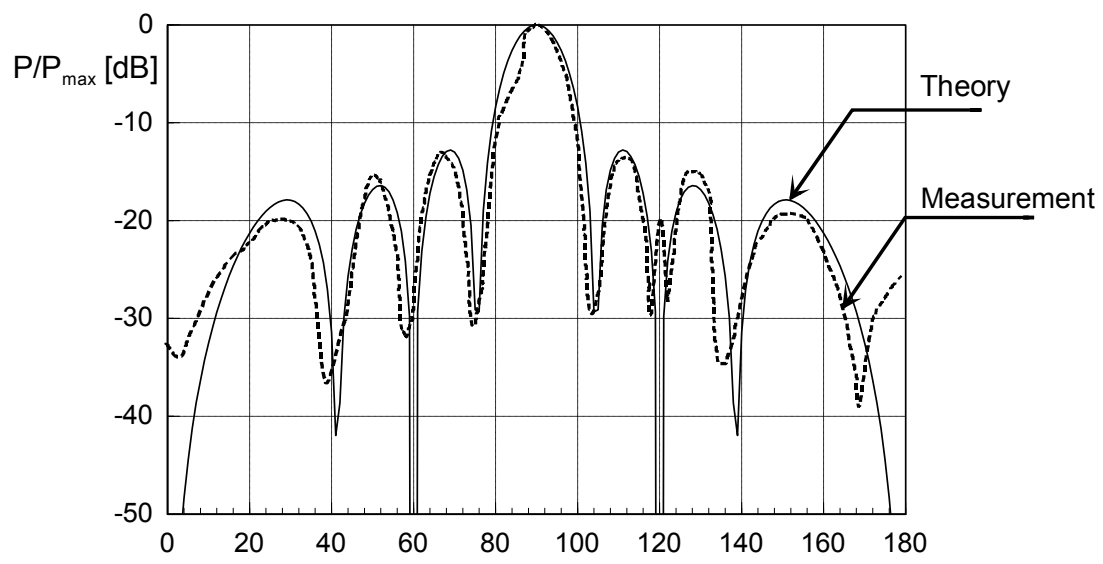

Figure 4: Radiation characteristic of antenna array.

\section{Conclusions}

Construction of antenna arrays placed on multilayer dielectric allows for the wanted modelling parameters and characteristics of the antenna array. An analysis of such a type of antennas which take into account the structure of the surface, true for each bandwidth, is based on the green function and moment method. The problem has been analysed, taking into account the distribution of field on Floquet harmonics, with usage of vector distribution on base functions. The proposed solution also allows for an analysis of antenna arrays with variable dimensions of a basic cell. It is a proposition of a precise and effective solution of problem of diagnosis on periodical antenna array with limited dimensions. One should stress, that a very important problem during solution is optimising the computer program in order to obtain maximum exactitude of calculations with minimal time needed.

\section{References}

[1] Hall P., James J.: "Handbook of microstrip antennas" Peter Peregrinus Ltd., London 1989

[2] Oltman G.H. "Electromagnetically coupled microstrip dipoles", IEEE Transactions on Antennas and Propagation AP-29, No 1, 1981.

[3] Pozar D., Tsay W.: "Application of the FDTD technique to periodic problems in scattering and radiation", IEEE Microwave and Guided Wave Letters, vol.3, August 1993. 
[4] Scott C.: „The spectral domain method in electromagnetics”, Artech House, London 1989.

[5] Hansen, Numerical Solution of Antennas in Layered Media, Wiley, New York, 1989.

[6] E. Munson "Conformal microstrip antennas and microstrip phased arrays" IEEE Trans. Antennas and Propagation vol. 281974 pp. 74- 78

[7] K. S. Yee "Numerical solution of initial boundary value problems involving Maxwell's equations in isotropic medias", IEEE Trans. Antennas and Propagation, vol. 14, 1966. pp. 302 - 307.

[8] C. J. Railton, E. M. Daniel, D. L. Paul, J. P. McGreen "Optimized absorbing boundary conditions handicap the analysis of planar circuits using the finite difference time domain method" IEEE Trans. Antennas and Propagation vol. 41, 1993 pp. 290 - 296

[9] Z. Bi, K. Wu, Ch. Wu, J. Litva "And dispersive boundary condition handicap microstrip component analysis using the FDTD method", IEEE Trans. Antennas and Propagation, vol. 40, 1992.pp. 774 - 777

[10] A. Taflowe "Advance in computational electrodynamics The Finite Difference Time Domain" Artech House Boston, 1995

[11] D. M. Sheen, S. M. Ali, M. D. Abouzahra, J. A. Kong, "Application of the three - dimensional finite difference time domain Method this the analysis of planar microstrip circuits " IEEE Trans. he Microwave Theory and Techniques, vol. 38, 1990 pp. 849-857

[12] Z. Bi, K. Wu, Ch. Wu, J. Litva, "Accurate characterization of planar printed antennas using finite difference time domain method" IEEE Trans. he Antennas and Propagation vol. 40 pp. 526-533

[13] A. Reinex, B. Jecko "Analysis of Microstrip Patch Anntenas Using Finite Difference Time Domain IEEE Trans. Antennas and Propagation, vol. 37, 1989. pp. 1361 - 1369. 